\title{
An Advanced Measurement System for Vortex-Induced-Vibrations Characterization in Large-Scale Risers
}

\author{
M. Ordonez*, M.O. Sonnaillon ${ }^{\dagger}$, D. Murrin*, N. Bose ${ }^{\ddagger}$, and W. Qiu* \\ * Memorial University of Newfoundland, St. John's, NL A1B 3X5 \\ ${ }^{\dagger}$ Balseiro Institute, National University of Cuyo, San Carlos de Bariloche, Argentina, 3168 \\ $\ddagger$ Australian Maritime Hydrodynamics Research Centre (AMHRC), Newnham, Tasmania, 7248
}

\begin{abstract}
This paper presents the development of an advanced measurement system for real-time characterization of Vortex Induced Vibrations (VIV) in large-scale marine risers. Marine risers play a critical role in offshore oil and gas operations by transporting mud, debris, and hydrocarbons from the reservoir to the surface platform. The mechanical structure of the riser is constantly subject to the effect of vortex shedding produced by seawater flow, which may result in severe oscillations known as VIV. At present, the prediction of this potentially destructive phenomenon is a challenging area of research that has gained special attention.

While there have been significant research contributions on vortex induced vibrations on small riser models $(8-10 \mathrm{~m}$ in length), the number of experiments on large-scale risers is limited, mainly due to technical complexity, logistics, and cost involved in large scale testing. Furthermore, the experiments that have been carried out are often proprietary and their publication in scientific literature has been limited. High mode results have been a challenge since it is generally accepted that at least five accelerometers are required to accurately map a cycle, and most of the riser models have been instrumented with no more than ten or twenty accelerometers due to the associated technical challenges. The advanced system described herein is modular and has been tested with sixty-four accelerometers, theoretically capable of mapping 25 modes of vibration. This is, therefore, a significant development towards understanding the vortex shedding phenomenon at high modes.

A complete description and design considerations for this advanced measurement system is presented in this paper. This includes aspects such as system architecture, hardware and software design, mounting procedure, and communication protocol for an actual application.
\end{abstract}

\section{INTRODUCTION}

The flow around circular cylinders is subject to vortex shedding and Vortex Induced Vibration (VIV) occurs when the vortex shedding frequency is approximately equal to the structure's natural frequency. High-mode VIV can occur with deepwater marine risers due to their excitation from current forces and high tension. There is a strong need to address deepwater issues on marine risers as offshore oil and gas exploration moves into ever increasing water depths.

In 1976, a VIV test system with 2 accelerometers mounted in a $23 \mathrm{~m}$ cable was presented [1]. This system capable of mapping 7 modes was held under constant tension in uniform flow. Using the same model, a similar test was performed by [2] in 1983 at a higher flow velocity using 7 pairs of accelerometers over the $23 \mathrm{~m}$ length of cable. Similarly to the results of [1], a maximum mode number of 7 was reported. A $267 \mathrm{~m}$ Kevlar cable using 3 accelerometers was employed to find that its response was similar to that of an infinite string [3]. In a different kind of arrangement, 6 pairs of accelerometers were mounted to a $17.7 \mathrm{~m}$ rubber hose and reported a maximum mode number of 11 [4]. Large scale tests involving two composite fiberglass pipes of $61 \mathrm{~m}$ and $122 \mathrm{~m}$ long with $33 \mathrm{~mm}$ in diameter were conducted [5]. A total of 24 tri-axial accelerometers spaced evenly along the cable were employed reporting a maximum of mode of 25 .

To date, high mode results from large scale VIV tests have been limited by the length to diameter ratio of models (the aspect ratio), cost, and the technical challenges associated with instrumenting them. The architecture of the measurement system presented in this work provides a relatively inexpensive, modular system that is capable of mapping much higher modes of vibration than has previously been possible and is a significant development towards understanding the vortex shedding phenomenon at high modes. A complete description and design considerations for this advanced measurement system is presented in this paper. This includes aspects such as system architecture, hardware and software design, mounting procedure, and communication protocol for an actual application.

\section{SySteM ARCHITECTURE}

A conceptual block diagram of the proposed system is shown in Fig. 1, where three main components can be identified: sensor terminals based on a Digital Signal Processors (DSP), synchronization module, and a supervisory computer. The sensor modules are distributed inside the test section pipe (i.e., polyethylene) while the synchronization module and computer remains outside (i.e., on the deck of ship). The core of the system is the computer and synchronization module, and the number of sensor modules is selected based on the length of the pipe and the number of mapping modes required for the experiment. As can be deduced from the block diagram in Fig. 1, the concept of modularity is one of the key features of the proposed measurement system. This modular architecture allows increasing the number of modules with the additional advantage of low cost per module. 


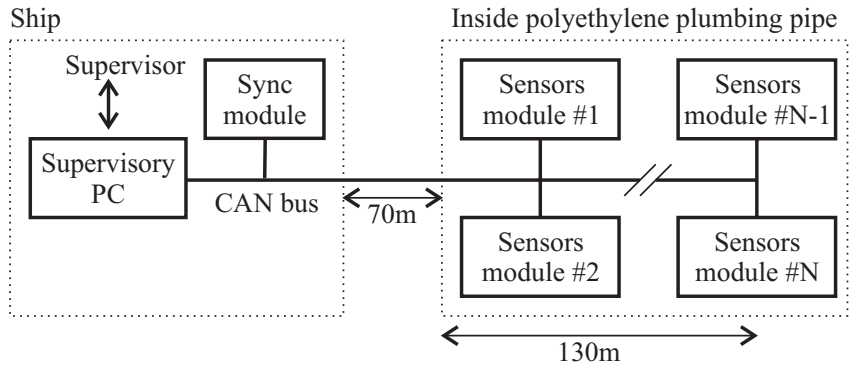

Fig. 1. Block diagram

The connection between the sensor modules, the synchronization module, and the computer is performed through an industrial communication bus that ensures robust high-speed data transmission even under noisy electrical environments. The system is coordinated by the synchronization module to ensure accurate simultaneous data acquisition. This is achieved by means of a triggering signal produced by the synchronization module, which is preprogrammed with the user interface (sampling rate and test duration). After each conversion, data packets containing motion information are transmitted in realtime through the communication bus. The role of the computer is to monitor and collect the data coming from the modules, and display the evolution of the riser with a user interface. What follows is a detailed description of the many components of the system.

\section{Sensor Modules}

A conceptual block diagram of the sensor module is presented in Fig. 2. The module is based on a fixed-point Digital Signal Processor (DSP) which performs data acquisition from the sensors and transmits information packages through a Control Area Network (CAN) bus. The selected hardware architecture provides the flexibility required to perform realtime digital signal processing within each module while maintaining low cost ( $\sim \$ 10$ per DSP chip). Precision instrumentation is used to interface the sensors and the Analog to Digital Converter (ADC) of the DSP. In order to supply the processor, signal conditioning, and sensors, the board requires unregulated $12 \mathrm{~V}$ to $7.5 \mathrm{~V}$ on its input terminals. As well, each sensor module is connected to the rest of the system through a shared communication bus (CAN) that has been physically implemented with a Category 5e network cable. Since only 2 wires are required for the CAN bus, the additional pairs are used to increase the reliability of the system as described in section IV.

A picture of the electronic module including acceleration, yaw gyro rate, and magnetic compass sensors is shown in Fig. 3 (referred to as full module in this work). Sensors which are not required can be eliminated from the board. In the application example, one full module is placed every 20 acceleration only modules. Hence, most of the modules in the system only account with a 3-axis accelerometer as the one shown in Fig. 4. The Printed Circuit Board (PCB) layout has been developed following strict design rules for

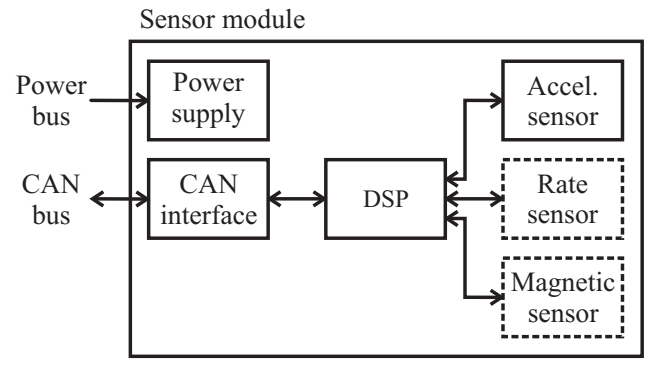

Fig. 2. Sensor module block diagram.

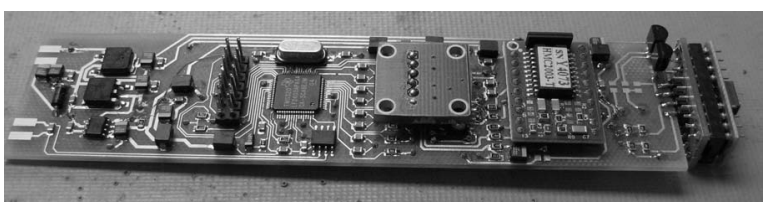

Fig. 3. Fully instrumented sensor module (acceleration, yaw gyro rate, and magnetic compass).

mixed signal circuits. These include split power supplies, split ground planes with LCL filtering, and cross-taking reduction techniques. The mechanical arrangement was designed to fit a PCV pipe as small as $40 \mathrm{~mm}$ of inner diameter. A mechanical adapter (placement puck) is employed to obtain the final desired diameter and alignment. Figure 5 shows a picture of a module and mechanical adapters for a $40 \mathrm{~mm}$ inner diameter pipe. As can be noted, the pucks not only hold the electronic board but also allow room for the power wires and communication cables. Since the processor that controls the sensor module has a small case (64TQFP package), the size of the board is inherently limited by the mechanical dimensions of the sensors mounted in the module (i.e., 3-axis magnetic sensor HMC2003).

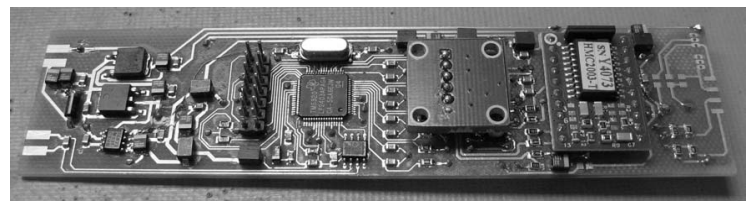

Fig. 4. Partially instrumented sensor module (acceleration only)).

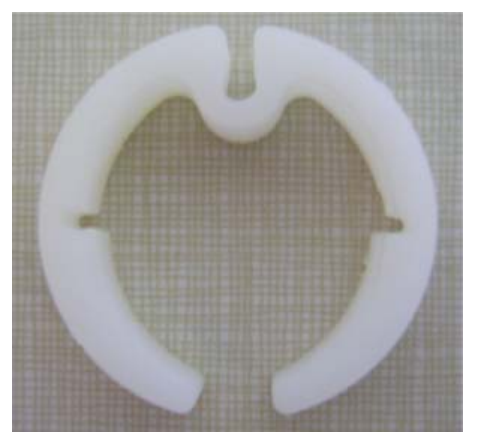

Fig. 5. 40mm Placement puck. 
When the measurement system is operated with a large riser model and several sensor modules, the dimensioning of the $\mathrm{dc}$ power bus wires should be carefully considered. The dc bus wire must be selected to ensure that the last module in the system is supplied with at least the minimum allowed voltage ( $7 \mathrm{~V}$ for the internal power supply of the sensor module). In order to calculate the voltage drop across the any point in the power distribution line, the following equation should be employed:

$$
V=\sum_{n=1}^{N} \sum_{k=n}^{N} I_{k} R_{k}
$$

where $N$ is the total number of modules, $I_{k}$ is the current consumption of module number $k$, and $R_{k}$ is the electrical resistance of the wire between modules $k$ and $k-1$. The first resistance, $R_{1}$, is the resistance of the wire between the power supply and the first module.

The electrical resistance of the wire is computed by using the copper resistivity $(\rho)$, the wire diameter $(d)$ and the distance between modules $(l)$ :

$$
R=\frac{\rho l}{d}
$$

The only variable for design process is the wire diameter, hence the AWG cable was selected to assure that the last module receive a high enough voltage. Thicker wires are more difficult to mount into the PVC pipe and also are heavier.

This highlights the importance of minimizing power consumption per module to avoid dealing with impractical wire sizes. As well, the concept of redundancy plays an important role in large scale instrumented risers. Therefore, two or more power lines are strongly recommended in such applications.

\section{SYNCHRONIZATION MODULE}

The synchronization module is an external subsystem that is preprogrammed by the computer prior to each test. In order to simplify the implementation and reduce the overall cost of the system, the synchronization module is an uninstrumented sensor module. The synchronization module runs a programmable precision timer that triggers the sensors modules simultaneously at the desired sampling rate. This is achieved by sending a periodical synchronization packet through the CAN bus. As a result of receiving the synchronization packet, the sensor modules perform data acquisition of the signal coming from the sensors (i.e. accelerometers). A picture of the synchronization module is presented in Fig. 6, in which both the computer (DB-9 connector) and the sensor modules (TIA/EIA-568-B connector) are connected to the sync module. A description of the principle of operation of the communication channel is presented in the following section.

\section{COMmunicAtion CHANNEL}

The communication channel selected for this system is the Controller Area Network, known as CAN bus. It was firstly designed for automotive applications by Bosch. Now it is the most used communication bus in commercial cars and it

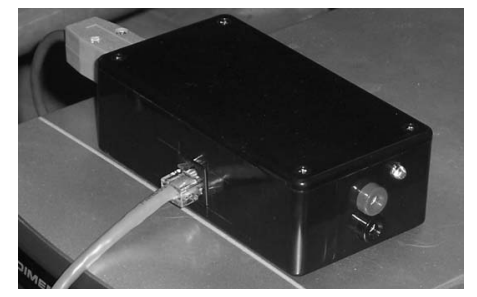

Fig. 6. Synchronization module.

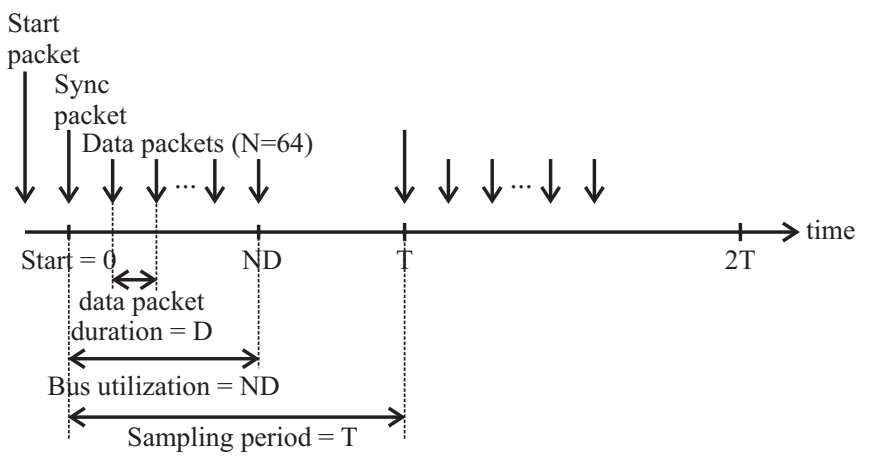

Fig. 7. Schematic diagram of the CAN bus timeline.

is being widely used in many other industrial applications. Its physical layer is based on a pair of copper wires. The communication protocol is implemented with the DSP on-chip CAN controller.

This bus was selected as the communication channel for this application because of the following characteristics:

1) High bandwidth: Its maximum $1 \mathrm{Mbps}$ (mega bits per second) allows a high number of modules (+64) acquiring at high sampling rates (i.e. more than $20 \mathrm{~Hz}$ ).

2) High data integrity: Its arbitration system and error checking protocol assures correct reception of data packets, even with high channel utilization.

3) Real time operation: Due to its arbitration system, the exact duration of each packet transmission can be predicted, hence the bus utilization can be optimally designed.

4) High robustness: CAN physical layer is designed to work under harsh electrical environments, keeping very low transmission error rates.

Although the communication bus has a high bandwidth, the data packet transmission must be properly organized in order to allow a high number of modules, operating at high sampling frequencies. The organization is based on assigning a different identification number (ID) to each module. This ID determines the relative time when each module sends its data. Figure 7 shows a simplified timeline of the CAN bus. The experimental test is started by the supervisory computer that sends a Start Packet. This packet is only received by the Sync Module, and has the information of the sampling period (T). Automatically, the Sync Module programs its internal timer and periodically sends the Sync Packets to the Sensor Modules. All the Sensor Modules receives almost at the same time the Sync Packet, 
and immediately they acquire the sensors data. Depending on its own ID, each Sensor Module waits for a certain time to send its Data Packet, therefore packet collisions are avoided, and the communication channel is optimally used.

The time that each Sensor Module has to wait until it sends its data packet, is a pre-computed value, and depends on the channel speed, the size of the packet to be sent, and the ID number:

$$
t=\frac{B}{f} I D
$$

where $t$ is the time that the module must wait to send its packet, $f$ is the bitrate in bits per second, and $B$ is the packed size in bits. The exact packet size can be computed by considering the whole packet structure. Each CAN packet has a certain number of fields that are used in its protocol, such as ID number, data size, error checking bits, and the data bits. For more information about the CAN packets structure, see [6].

The CAN network is physically implemented with an Ethernet category $5 \mathrm{e}$ cable. In order to increase the reliability of the system all four pair of wires are employed to provide redundancy to the system. This conforms 4 communication lines in which only $1 / 4$ of the communication modules are connected to each line (one every four sensors). Upon a failure of one or more stranded pairs, the system would still account for sensor modules spanning the entire length of the riser. As well, in the unlikely event of failure of a CAN driver chip (short circuit) within a board, this arrangement allows the disconnection of the line associated with the problem. Redundancy is extremely important in large scale instrumented risers not only due to the significant amount of time required to mount or insert the measurement system in the pipe but also the complex process of field deployment. More details on mechanical and mounting aspects of largescale model riser can be found in [7].

\section{USER INTERFACE AND SyStem SPECIFICATIONS}

A standard personal computer is used to control and monitor in real-time the measurement system. It is connected to the CAN bus through a National Instruments PCI board. The user interface developed as part of the system runs under Windows operating system. It provides three basic functions: (1) parameters configuration, (2) real-time system supervision and (3) data visualization, as shown in Fig. 8.

The configuration section allows setting the sampling period, the total test time and the self-test modes. These self-test modes are used to check the correct operation of the sensor modules. A supervising feature reports the CAN bus activity in real-time. When faults or operation problems arise, the user is immediately informed to proceed with a troubleshooting procedure. The data visualization is also part of the interface allowing to plot the measurements as a function of time. As well, it allows selecting individual modules and zooming of certain areas of the plot. Table I presents a list of important specifications of the system.

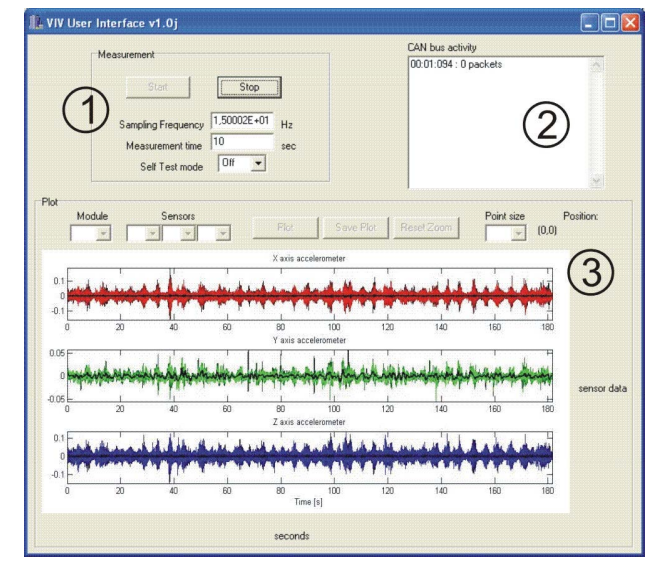

Fig. 8. User interface software screenshot.

TABLE I

SYSTEM SPECIFICATIONS

\begin{tabular}{|c|c|}
\hline Characteristic & Specification \\
\hline $\begin{array}{l}\text { Maximum communication } \\
\text { channel bandwidth }\end{array}$ & $1 \mathrm{Mbps}$ \\
\hline $\begin{array}{l}\text { Maximum number of sensor modules } \\
\text { sensor modules }\end{array}$ & $\begin{array}{l}\text { Only limited by } \\
\text { channel bandwidth }\end{array}$ \\
\hline Processor processing power & 40 MIPS, 16 bit \\
\hline $\begin{array}{l}\text { Accelerator full-scale range } \\
\qquad(3 \text { axes })\end{array}$ & $3.6 \mathrm{~g}$ \\
\hline Gyro full-scale range & $300 \% / \mathrm{s}$ \\
\hline $\begin{array}{l}\text { Magnetic sensor full-scale range } \\
\text { (3 axes) }\end{array}$ & 2 gauss \\
\hline $\begin{array}{l}\text { Analog to digital converter } \\
\text { resolution (for the } 3 \text { sensors) }\end{array}$ & 10 bit \\
\hline Supply voltage & $12 \mathrm{~V}-7 \mathrm{~V}$ \\
\hline Mechanical dimension & $35 \mathrm{~mm} \times 15 \mathrm{~mm} \times 85 \mathrm{~mm}$ \\
\hline
\end{tabular}

\section{CONCLUSION}

The development of an advanced measurement system for Vortex-Induced-Vibrations has been presented in this work. Real-time data monitoring, flexibility, and modularity are the main features of the proposed system. The proposed architecture has low cost and allows on-board signal processing, real-time data transmission and high precision in the instrumentation. Furthermore, the system can be fully configured by software. The sampling rate and number of sensors can be optimized to reach the particular requirements of each experiment. The user interface software allows an easy configuration of the system, real-time monitoring of the CAN bus activity and simple visualization of the acquired data.

\section{ACKNOWLEDGMENT}

The authors would like to thank the Natural Sciences and Engineering Research Council (NSERC) of Canada and Petroleum Research Atlantic Canada (PRAC) for their financial contributions. Furthermore, the in-kind contributions of the National Research Council Canada-Institute for Ocean 
Technology, Oceanic Consulting Corporation, and Chevron Energy Technology Company are greatly appreciated. Finally, the authors would like to thank the contributions of Chris Batten and Roy Crocker (MUN Technical Services) towards the development of the system and the design advice supplied by Charles Humphrey is gratefully acknowledged.

\section{REFERENCES}

[1] J. Vandiver, and C. Mazel, "A field study of vortex-excited vibrations of marine cables," Proceedings of the 1976 Offshore Technology Conference, Paper NO. 2491, Dallas, 1976.

[2] J. Vandiver, "Drag Coefficients of long flexible cylinders," Proceedings of the 1983 Offshore Technology Conference, PaperNO. 4490 Houston, 1983.

[3] Y. Kim, and J. Vandiver, "Vortex-Induced Vibration and Drag Coefficients of Long Cables Subjected to Sheared Flow," Proceedings of the 4th OMAE Symposium, Volume One, ASME, Dallas, Texas, February 1985

[4] J. Vandiver, and T. Chung, "Predicted and measured response of flexible cylinders in sgeared flow," Proceedings of ASME Annual Meeting Symposium on Flow-Induced Vibration, Chicago, 1988.

[5] J. Vandiver, H. Marcollo, S. Swithenbank, and V. Jhingran, "High mode number vortex-induced vibration field experiments," Proceedings of the 2005 Offshore Technology Conference, Paper No. 17383, Houston, 2005.

[6] W. Lawrenz, "CAN System Engineering," Springer,1997, pp. 26-30, 82101 .

[7] D. Murrin, M. Ordonez, G. Stone, N. Bose, and W. Qiu, "High Mode Vortex Induced Vibration (VIV) Experiments on a Large-Scale Riser," Proceedings of the 2007 IEEE OCEANS, Vancouver, 2007. 